\title{
Solid Phase Thermal Polymerization of Macrocyclic Bisphenol A Carbonate Tetramer Using Bisphenol A as Initiator
}

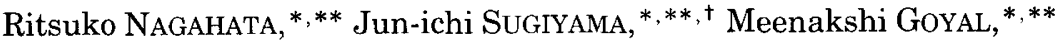 \\ Michihiko ASAI, ${ }^{*, * *}$ Mitsuru UEdA, ${ }^{*, * *, * * *}$ and Kazuhiko TAKEUCHI ${ }^{*, * *}$ \\ * Joint Research Center for Precision Polymerization-Tsukuba, \\ 1-1 Higashi, Tsukuba, Ibaraki 305-8565, Japan \\ ** National Institute of Materials and Chemical Research, \\ 1-1 Higashi, Tsukuba, Ibaraki 305-8565, Japan \\ *** Tokyo Institute of Technology, Meguro-ku, Tokyo 152-8552, Japan
}

(Received March 27, 2000; Accepted June 20, 2000)

\begin{abstract}
Solid phase thermal ring-opening polymerization of macrocyclic bisphenol A carbonate tetramer using bisphenol A as initiator was investigated. To find optimum conditions for the polymerization, reactions were performed with various concentrations of bisphenol A at different temperatures and times. Under optimized conditions, colorless polymer with ultra high molecular weight $\left(M_{\mathrm{w}}>1000000\right)$ was produced in a short time. Terminal functionalities of the polymers were investigated with the help of matrix-assisted laser disorption ionization time-of-flight (MALDI-TOF) mass analysis.

KEY WORDS Polycarbonate/Macrocyclic Oligomer/Ring-Opening Polymerization/Thermal Polymerization / Solid Phase Polymerization / Bisphenol A /
\end{abstract}

Aromatic polycarbonate is a well-known polymer with good properties, particularly such as impact resistance, electrical properties, optical clarity, dimensional rigidity and the like. Because of these special qualities, aromatic polycarbonate is widely used as the most representative engineering plastic. ${ }^{1}$ Bisphenol A polycarbonate is generally produced by; (1) transesterification of diphenylcarbonate with bisphenol A and (2) homogeneous or interfacial polycondensation of phosgene with bisphenol A. In either method, it is very difficult to remove residual impurities such as catalysts for transesterification and/ or salts, completely. These impurities may cause drop in electrical and optical properties, and accelerate and/or initiate degradation of the polymer during melt processing.

Brunelle $e t a l .^{2}$ reported that polymerization of bisphenol A macrocyclic oligocarbonates mixture (dimer to henicosamer) with catalysts gave polymers of higher molecular weight $\left(M_{\mathrm{w}}=50000-300000\right)$ than those synthesized by transesterification or phosgene method. This cyclic oligomer mixture has fluidity near its melting point, i.e., around $200^{\circ} \mathrm{C}$ and therefore can be polymerized in the molten phase. Although reactive processing of lowviscosity small-ring monomers is well-established in more conventional areas of polymer chemistry, in most of these cases, oligomeric mixtures of the macrocyclics are used as starting materials to reduce melting points below usable reaction temperatures. ${ }^{3-6}$

Since melting points of macrocyclic bisphenol A carbonate dimer (c-2mer), trimer (c-3mer), and tetramer (c-4mer) were reported to be very high $\left(>330^{\circ} \mathrm{C}\right)$, melt polymerization of each seems difficult. ${ }^{2 b, 7}$ Recently, we found macrocyclic bisphenol A carbonates with small ring sizes (c-2mer to c-4mer) can undergo ring-opening polymerization without any initiator in solid phase at the temperatures below melting points to give ultra high

\footnotetext{
${ }^{\dagger}$ To whom correspondence should be addressed.
}

molecular weight polymers $\left(M_{\mathrm{w}} 2000000\right){ }^{8}$ Remnants of additives or impurities in the polymer are undesirable from an industrial point of view, because they might cause degradation or deterioration of materials during processing. This method is free of such disadvantages as no additives such as catalysts are added. The c-4mer is the major content of the cyclic mixture obtained by present synthesis. Reactivity for ring-opening polymerization (without initiator) is relatively low in comparison with the c-2mer and the c-3mer. Therefore, it is important to investigate the possibility of enhancing the reaction rate of uniform c- 4 mer. Although one possible approach is the addition of certain catalyst/initiator which accelerates the ring-opening of comparatively stable c-4mer, residue of metal or halogen additives in the products might spoil the advantages. However, if bisphenol $\mathrm{A}$ is used as initiator for the ring-opening polymerization of c-4mer, it will not remain as undesired impurity since it reacts and become a part of the polymer chain. Therefore, solid phase thermal polymerization of the c-4mer using bisphenol A as initiator was investigated.

\section{EXPERIMENTAL}

\section{Materials and Measurements}

Macrocyclic oligomer mixture (c-2mer to c-21mer) was prepared by hydrolysis/condensation reaction and c-4mer was isolated from the mixture as reported in the literature. ${ }^{8,9}$ Repetitive recrystallization from $p$-xylene afforded transparent plates. The crystals occluding $p$-xylene ${ }^{9}$ were easily removed by drying under vacuum at $70^{\circ} \mathrm{C}$ overnight. Preparation of completely uniform and solvent free c-4mer was confirmed by ${ }^{1} \mathrm{H}$ nuclear magnetic resonance (NMR), ${ }^{13} \mathrm{C} \mathrm{NMR}$, electrospray ionization time-of-flight (ESI-TOF) mass spectrometry, matrix-assisted laser disorption ionization time-of-flight (MALDI-TOF) mass spectrometry, size exclusion chrom atography (SEC), and X-Ray crystallographic analysis. E 


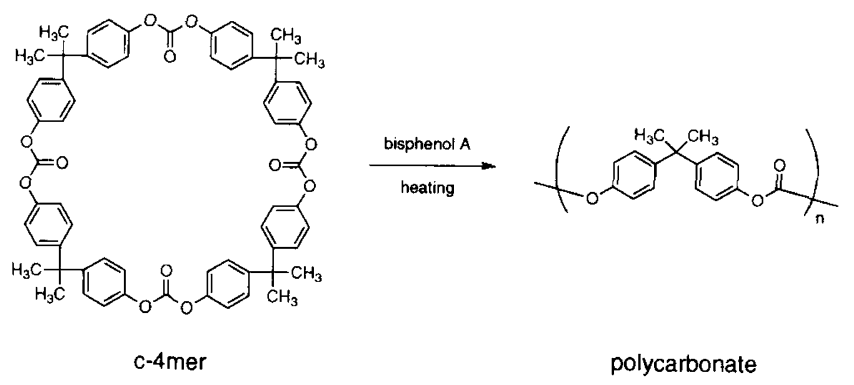

Scheme 1.

SI-TOFMS: calcd for $\mathrm{C}_{64} \mathrm{H}_{57} \mathrm{O}_{12}$ (monoisotopic), 1017.385 $[\mathrm{M}+\mathrm{H}]^{+} \mathrm{m} / \mathrm{z}$; found, $1017.401[\mathrm{M}+\mathrm{H}]^{+} \mathrm{m} / \mathrm{z}$. Results of other analyses have been reported elsewhere separately. ${ }^{8 b, 9}$ Bisphenol A (2,2-bis(4-hydroxyphenyl)propane) was recrystallized from chlorobenzene. Bisphenol Z (1,1-bis(4-hydroxyphenyl)cyclohexane) was recrystallized from ethanol/water.

\section{Instruments}

An ESI-TOF mass spectrometer (PE Biosystems Mariner) was used for molecular weight analysis of c-4mer. SEC analysis was performed with a JASCO GULLIVER system coupled with Shodex K-407L, K-804L, or KF-802 columns and an ultraviolet detector set at $254 \mathrm{~nm}$. The mobile phase was chloroform and data were recorded at $35^{\circ} \mathrm{C}$ at a flow rate of $0.3 \mathrm{~mL} \mathrm{~min}^{-1}$. Molecular weights and molecular weight distributions were estimated by comparison with polystyrene standards. A MALDITOF mass spectrometer (Shimadzu/Kratos Kompact MALDI III) was used for molecular structure analysis of the polymerization products using 2,5-dihydroxybenzoic acid as a matrix, and tetrahydrofrane as solvent.

\section{Ring-Opening Polymerization of Macrocyclic Bisphenol A Carbonate Tetramer (C-4mer)}

Polymerization reaction was carried out by heating c-4mer under nitrogen with initiators (bisphenol A or bisphenol $Z$ ) on a programmed heating device (Seiko Instruments Inc. SSC5100). C-4mer and initiators were dissolved in chloroform at specified concentrations (mol \% bisphenol A carbonate repeat unit). After removing the solvent by a rotary evaporator, the starting material was completely dried in vacuo at $70^{\circ} \mathrm{C}$ overnight. Finely ground starting materials $(1.3-1.6 \mathrm{mg})$ were placed on the aluminum pan with cover and heated from $20^{\circ} \mathrm{C}$ to the holding temperature at $10^{\circ} \mathrm{C} \mathrm{min}^{-1}$, and held there for the required time. Conversion percentage and molecular weight of the polymer were determined by SEC.

\section{RESULTS AND DISCUSSION}

Macrocyclic tetramer (c-4mer) mixed with bisphenol A in chloroform was dried and was ground as fine as possible using a pestle and mortar. The ground powder was kept in a washed and dried aluminum pan generally used for DSC analysis. Thermal polymerization was carried out on a programmed heating device. Pan was heated from $20^{\circ} \mathrm{C}$ to the required temperature

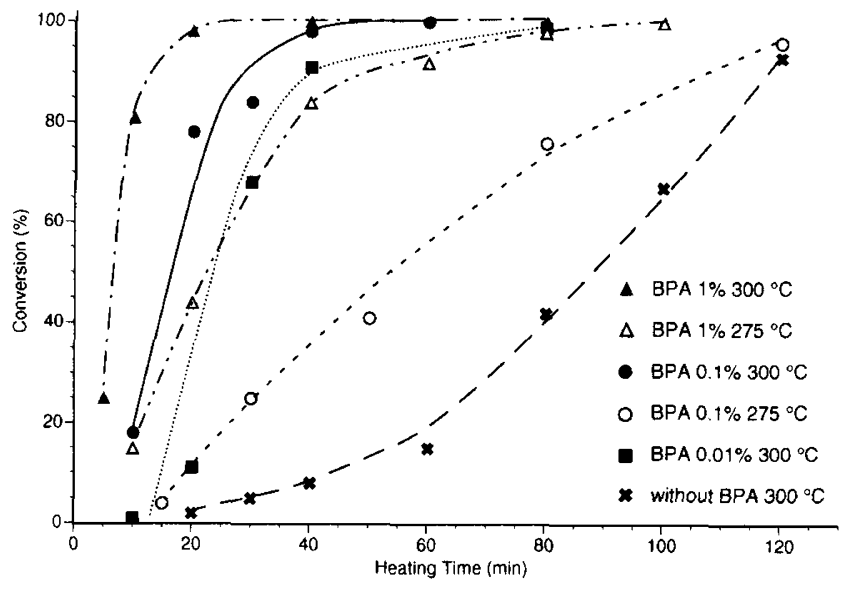

Figure 1. Effects of polymerization time at various concentrations of bisphenol A (BPA) on conversion.

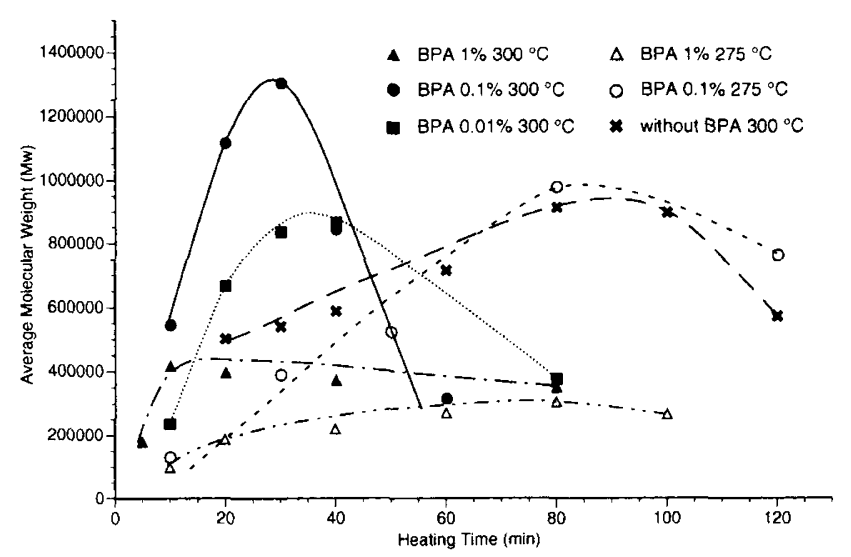

Figure 2. Effects of polymerization time at various concentrations of bisphenol A (BPA) on average molecular weight.

$10^{\circ} \mathrm{C} \min ^{-1}$ in a furnace under dry nitrogen atmosphere. Melting point of c-4mer is reported to be very high (368 $\left.-372^{\circ} \mathrm{C}\right){ }^{2 \mathrm{~b}}$ In the present work, since polymerization was carried out at temperatures much lower than the melting point, and the products obtained were white powdery solid even at high conversions, the reaction surely must have proceeded in the solid phase. All products obtained by heating c-4mer with bisphenol A were soluble in chloroform and molecular weights were estimated by SEC. The results of polymerization are summarized in Figures 1 and 2.

Heating c-4mer with $0.01 \mathrm{~mol} \%$ bisphenol A for $20 \mathrm{~min}$ at $300^{\circ} \mathrm{C}$ under nitrogen produced a white colorless polymer with $M_{\mathrm{w}} 665700\left(M_{\mathrm{w}} / M_{\mathrm{n}} 2.8\right)$ in $11 \%$ yield. At reaction time of $40 \mathrm{~min}, 91 \%$ of c- 4 mer was converted to colorless polymer of $M_{\mathrm{w}} 865700\left(M_{\mathrm{w}} / M_{\mathrm{n}} 3.1\right)$. Polymerization without initiator at the same temperature resulted in polymers of $M_{\mathrm{w}} 501600$ in $2 \%$ yield in $20 \mathrm{~min}$ and $M_{\mathrm{w}}$ 588300 in $8 \%$ yield in 40 min (Figure 1). ${ }^{8 b}$ Bisphenol A thus assists in making the ring opening easier and thereby helping polymerization in the solid phase. At $80 \mathrm{~min}$ in the presence of $0.01 \mathrm{~mol} \%$ bisphenol A, increase in conversion percentage was observed (99\%) but molecular weight decreased to 376900 , probably due to degradation caused by unreacted bisphenol A.

To see the effects of catalysis concentration on poly- 
mer yield and molecular weight, bisphenol A concentration was increased to $0.1 \mathrm{~mol} \%$. The polymerization became faster and $84 \%$ conversion was achieved within $30 \mathrm{~min}$ at $300^{\circ} \mathrm{C}$ giving an ultra high molecular weight, colorless polymer of $M_{\mathrm{w}} 1302000\left(M_{\mathrm{w}} / M_{\mathrm{n}} 3.2\right)$. Complete conversion was finally attained in $60 \mathrm{~min}$. However, further increase in reaction time led to decrease in molecular weight, due to decomposition on prolonged heating. Keeping bisphenol A concentration constant, when the reaction temperature decreased to $275^{\circ} \mathrm{C}, 76 \%$ of monomer was converted to colorless polymer of $M_{\mathrm{w}} 979500$ $\left(M_{\mathrm{w}} / M_{\mathrm{n}} 3.2\right)$ in $80 \mathrm{~min}$. Further increase in reaction time to $120 \mathrm{~min}$ led to increase in conversion to $96 \%$ and no significant decrease in $M_{\mathrm{w}}$ was observed.

On increasing bisphenol A concentration to $1 \mathrm{~mol} \%$, polymerization rate became much faster. $81 \%$ conversion was achieved within $10 \mathrm{~min}$ at $300^{\circ} \mathrm{C}$ giving colorless polymer of $M_{\mathrm{w}} 416900\left(M_{\mathrm{w}} / M_{\mathrm{n}} 3.0\right)$. At this temperature and initiator concentration, higher molecular weight polymer could not be prepared in spite of prolonged reaction time. Keeping bisphenol A concentration at $1 \mathrm{~mol} \%$, when temperature was reduced to $275^{\circ} \mathrm{C}$, reaction rate reflected by conversion $v s$. time curve (Figure 1) was comparable to that under all other reaction conditions but polymer molecular weight was relatively lower $\left(M_{\mathrm{w}}<306100\right) .1 \mathrm{~mol} \%$ of initiator concentration is thus not suitable for preparation of ultra high molecular weight polymer.

SEC traces of polymers obtained in different reaction times exhibited only two separate peaks; one for the starting material (c-4mer) and the other for the polymer. With increased reaction time, as polymerization proceeded, polymer peak area kept increasing, while the area of peak corresponding to c-4mer kept decreased. This indicates that polymerization proceeds through chain reaction, and not through typical polycombination reaction.

As demonstrated in Figure 1, polymerization rate of c-4mer in the absence of initiator was very slow in the initial stages of the reaction. This may be explained assuming the amount of active species such as terminal phenol type structures to be very low at the initial stage. However, slow thermal decomposition of c-4mer generates active species continuously, leading to active species accumulation on increasing reaction time, resulting in accelerated polymerization rate in later stages. The time-conversion relationship in the presence of the bisphenol A showed that c-4mer was consumed rapidly (Figure 1), suggesting that bisphenol A behaves as an initiator for ring-opening polymerization of c-4mer resulting in fast reaction rate right from the beginning.

Regarding molecular weight distribution $\left(M_{\mathrm{w}} / M_{\mathrm{n}}\right)$, the polymers obtained by heating with bisphenol A had narrower molecular weight distribution than by no-initiator reaction. On comparing polymerization rates of bisphenol $\mathrm{A}$ initiated reaction and no-initiator reaction, starting material as well as product are shown subjected to longer heating times in no-initiator reactions for obtaining high conversions ( $c a .90 \%$ ). Longer reaction time leads to more thermo-degradation along with polymerization, which broadens the molecular weight distribution in no-initiator reaction.

To investigate terminal functionalities of the polymer chains, MALDI-TOF mass analysis was carried out. The ultra high molecular weight of the polymer obtained in the present work was out of the permissible mass range of the MALDI analysis. Therefore, low molecular weight polymer was prepared separately by polymerization with high concentration of bisphenol A. Heating c-4mer with $5 \mathrm{~mol} \%$ bisphenol A for $15 \mathrm{~min}$ at $275^{\circ} \mathrm{C}$ produced a colorless and clear polymer with $M_{\mathrm{w}} 44400\left(M_{\mathrm{w}} / M_{\mathrm{n}} 1.9\right)$ in $95 \%$ yield. This low $M_{\mathrm{w}}$ polymer was not found suitable for detecting molecular-related ions and sufficient peaks could not be observed. By MALDI-TOF mass for molecular mass measurements should be limited to polymers with narrow molecular weight distributions because lighter molecules (outnumbering large ones) tend to saturate the detector, to suppress the detection of the later-arriving larger molecules. ${ }^{10}$ To solve this problem, a nearly monodisperse sample was prepared by repetitive fractionation using analytical SEC. Weight average molar mass $\left(M_{\mathrm{w}}\right)$ of the collected fraction was 6100 with dispersity of 1.1 (estimated by SEC based on standard polystyrene). Although a good MALDI spectrum was not obtained for the unfractionated sample, fractionated narrow disperse sample could be analyzed easily. Figure 3 shows MALDI-TOF mass spectrum of the fraction with $M_{\mathrm{w}} 6100$. The spectrum exhibited a single series of ion peaks at $\mathrm{m} / \mathrm{z} 254.3 n+251.3$ where 254.3 is the mass of repeat unit, $n$ number of repeat units, and 251.3, mass of end group and sodium cation. These values are consistent with those of sodium cationaised polycarbonate oligomeric species containing - $\mathrm{OH}$ group at both ends. Average molar mass $\left(M_{\mathrm{w}}\right)$ determined by the MALDI spectrum was 3667 and $M_{\mathrm{w}} / M_{\mathrm{n}}, 1.03$.

To demonstrate that bisphenol A reacts to become part of a polymer chain and not remain as a residual impurity, polymerization was carried out with bisphenol $\mathrm{Z}$ instead of bisphenol A. The polymer thus obtained was characterized by MALDI-TOF mass analysis. To obtain a model polymer with very low molecular weight for analysis by MALDI, and understand the reaction mechanism in the initial stage of heating, large amount of bisphenol $\mathrm{Z}$ was added and heating was carried out for short time. C-4mer with $10 \mathrm{~mol} \%$ bisphenol $\mathrm{Z}$ was heated from $20^{\circ} \mathrm{C}$ to $250^{\circ} \mathrm{C}$ at $10^{\circ} \mathrm{C} \mathrm{min}$ min $^{-1}$ and cooled immediately. A colorless oligomer was obtained in $42 \%$ yield. The MALDI spectrum of the polymer fractionated by analytical SEC $\left(M_{\mathrm{w}} 4000, M_{\mathrm{w}} / M_{\mathrm{n}} 1.2\right)$ exhibited the insertion of as many as four bisphenol $\mathrm{Z}$ units per polymer chain (Figure 4). The bisphenol $\mathrm{Z}$ unit in the oligomer fraction confirms the additive bisphenol initiator react and became part of the main chain of the product. The phenoxide moiety attacked the carbon atom of the carbonate group of the c-4mer and ester-phenol transesterification took place. More than one bisphenol $\mathrm{Z}$ unit in one oligomer chain indicates frequent ester-phenol transesterification between bisphenol Z-linear oligomer and/or between linear oligomer-linear oligomer. The phenol moiety may thus react not only with c-4mers but also the produced linear oligomers. Conformational stress of the c-4mer as well as linear oligocarbonate may be low, and thus the active species react both with c-4mer and linear oligomer. 


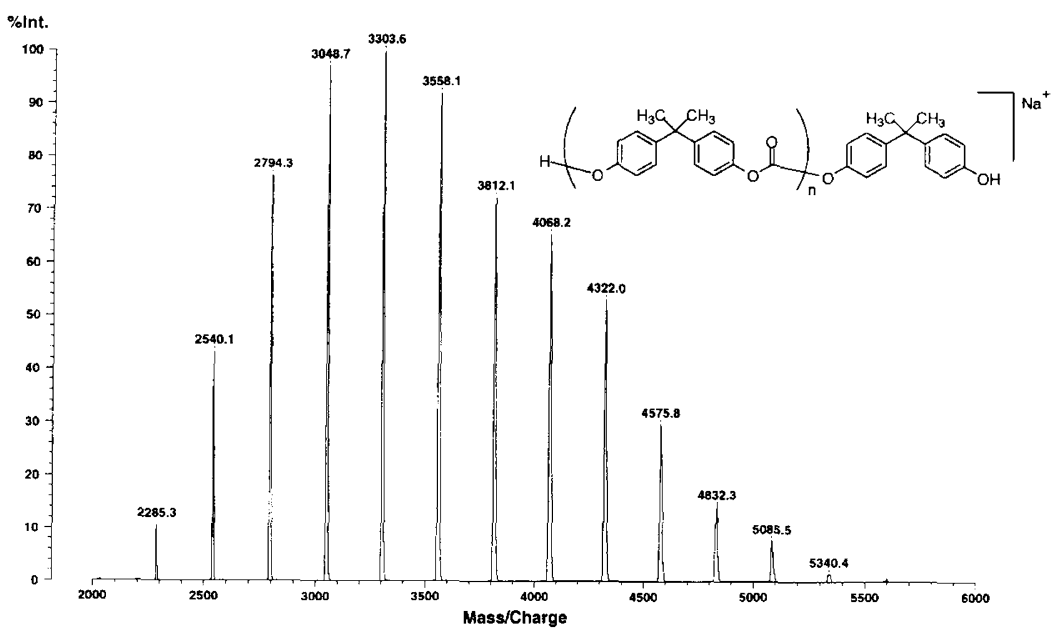

Figure 3. MALDI-TOF mass spectrum of the polymerization product initiated by bisphenol A.

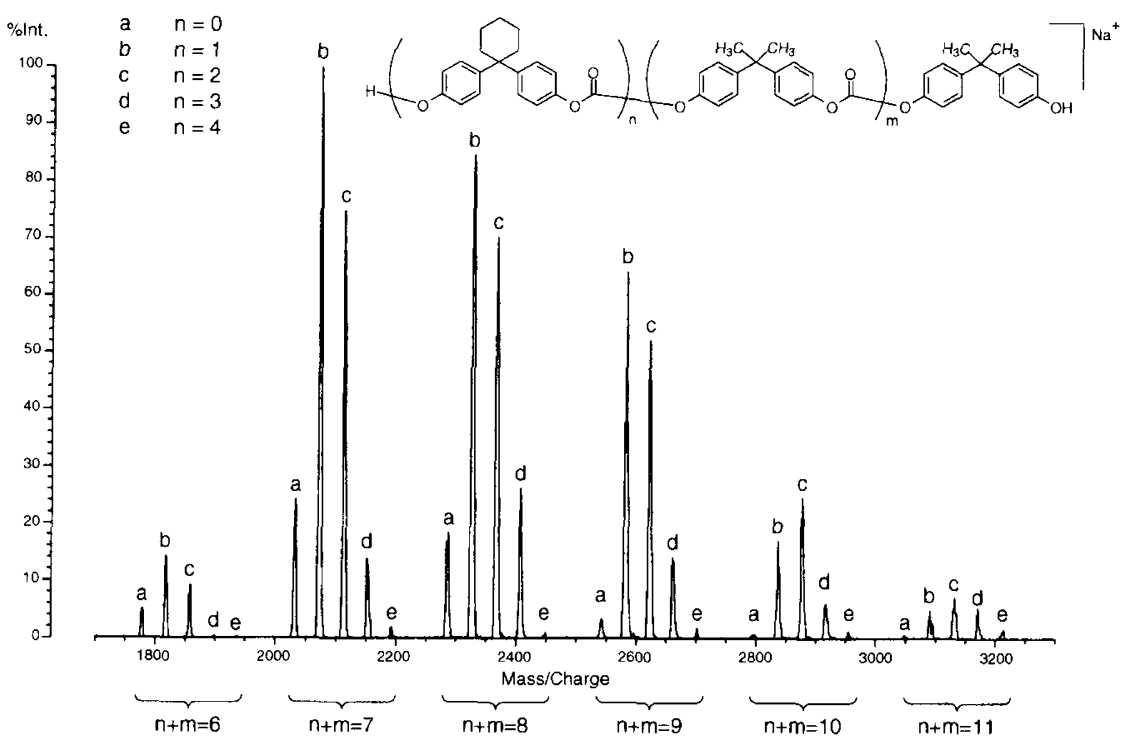

Figure 4. MALDI-TOF mass spectrum of the polymerization product initiated by bisphenol Z.

\section{CONCLUSIONS}

Thermal polymerization of macrocyclic bisphenol A carbonate tetramer (c-4mer) was carried out in the presence of various amounts bisphenol $\mathrm{A}$ as initiator at different temperatures and times. Reaction at $300^{\circ} \mathrm{C}$ with $0.1 \mathrm{~mol} \%$ bisphenol A resulted in an ultra high molecular weight polymer $\left(M_{\mathrm{w}}>1000000\right)$. Powdery and colorless appearance of the polymer confirmed the reaction to proceed in the solid phase. Bisphenol A thus makes ringopening of c-4mer much easier by generating active species in the initial stages of the reaction. This polymerization is environmental friendly because of no solvent, byproduct formation, or remnant catalytic impurities. Ultra high molecular weight polymer has excellent resistance against scratch and abrasion. The polycarbonate obtained by this method should prove useful as a new material for high performance application.
Acknowledgments. This work is a part of a project in Technology for Novel High-Functional Materials in Industrial Science and Technology Frontier Program, AIST, MITI.

\section{REFERENCES}

1. D. Freitag, G. Fengler, and L. Morbizer, Angew. Chem. Int. Ed., 30, 1598 (1991).

2. a) D. J. Brunelle and T. G. Shannon, Macromolecules, 24, 3035 (1991). b) D. J. Brunelle, E. P. Boden, and T. G. Shannon, J. Am. Chem. Soc., 112, 2399 (1990).

3. a) K. P. Chan, Y. Wang, and A. S. Hay, Macromolecules, 28, 653 (1995). b) K. P. Chan, Y. Wang, A. S. Hay, X. L. Hronowski, and R. J. Cotter, Macromolecules, 28, 6705 (1995). c) Y. Ding and A. S. Hay, Macromolecules, 29, 3090 (1996). d) Y. Wang, K. P. Chan, and A. S. Hay, J. Appl. Polym. Sci., 59, 831 (1996). e) Y. Wang, K. P. Chan, and A. S. Hay, Macromolecules, 29, 3717 (1996). f) Y. Wang, K. P. Chan, and A. S. Hay, J. Polym. Sci., Polym. Chem. Ed., 34, 375 (1996). g) C. Gao and A. S. Hay, Polymer, 36, 4141 (1995). h) Y. Wang, M. 
Paventi, K. P. Chan, and A. S. Hay, J. Polym. Sci., Polym. Chem. Ed., 34, 2135 (1996).

4. D. Xie, Q. Ji, and H. W. Gibson, Macromolecules, 30, 4814 (1997).

5. a) P. Hubbard, W. J. Brittain, W. J., Jr. Simonsick, and C. W., III. Ross, Macromolecules, 29, 8304 (1996). b) P. Hubbard, W. J. Brittain, W. L. Mattice, and D. J. Brunelle, Macromolecules, 31, 1518 (1998). c) D. J. Brunelle, J. E. Bradt, J. SerthGuzzo, T. Takekoshi, T. L. Evans, E. J. Pearce, and P. R. Wilson, Macromolecules, 31, 4782 (1998). d) D. J. Brunelle and J. Serth-Guzzo, Polym. Prepr., Am. Chem. Soc., Div. Polym. Chem., 40(1), 566 (1999).

6. a) Y. H. Kim, J. Calabrese, and C. McEwen, J. Am. Chem. Soc., 118, 1545 (1996). b) Y. Wang and A. S. Hay, Macromolecules, 29, 5050 (1996). c) Y. Wang, K. P. Chan, and A. S. Hay,
Macromolecules, 28, 6371 (1995). d) Y. Ding and A. S. Hay, Macromolecules, 29, 4811 (1996).

7. H. Schnell and L. Bottenbruch, Makromol. Chem., 57, 1 (1962).

8. a) J. Sugiyama, R. Nagahata, M. Goyal, and K. Takeuchi, Kobunshi Ronbunshu, 54, 749 (1997). b) R. Nagahata, J. Sugiyama, M. Goyal, M. Asai, M. Ueda, and K. Takeuchi, Polym. Adv. Technol., 11, 294 (2000).

9. R. Nagahata, J. Sugiyama, M. Goyal, M. Goto, M. Asai, M. Ueda, and K. Takeuchi, Polym. Adv. Technol., 11, 294 (2000).

10. a) G. Puglisi, F. Samperi, S. Carroccio, and G. Montaudo, Rapid. Commun. Mass. Spectrom., 13, 2260 (1999). b) G. Puglisi, F. Samperi, S. Carroccio, and G. Montaudo, Rapid. Commun. Mass. Spectrom., 13, 2268 (1999). 